\section{Preventing transmission of infectious agents in the pediatric in-patients hematol- ogy-oncology setting: what is the role for non- pharmacological prophylaxis?}

Désirée Caselli, 1 Simone Cesaro,2 Susanna Livadiotti, 3 Ottavio Ziino, 4 Olivia Paolicchi, 1 Giulio Zanazzo, 5 Giuseppe M. Milano, 6 Maria Licciardello, 7 Angelica Barone, ${ }^{8}$ Monica Cellini, 9 De Santis Raffaella, ${ }^{10}$ Mareva Giacchino, ${ }^{11}$ Mario Renato Rossi,12 Maurizio Aricò, Elio Castagnola ${ }^{13}$ for the Infection Study Group of the Associazione Italiana Ematologia Oncologia Pediatrica (AIEOP)

1Dipartimento Oncoematologia

Pediatrica, Azienda Ospedaliero-

Universitaria Meyer, Firenze;

2Oncoematologia Pediatrica,Verona;

3UO Immunoinfettivologia, DMP

Ospedale Pediatrico Bambino Gesù

Roma; 4U.O. di Oncoematologia

Pediatrica, ARNAS Ospedali Civico, G. Di

Cristina e Benfratelli; 5U.O. Emato

Oncologia Istituto per I'Infanzia, Trieste;

'Unit of Pediatric Oncology and

Haematology, Ospedale S. Maria della

Misericordia, A.O. di Perugia; ${ }^{7}$ Centro di

Riferimento Regionale di Emato-

Oncologia Pediatrica Azienda

Ospedaliero Universitaria Policlinico

Gaspare Rodolico Catania; ${ }^{8}$ Pediatria e

Oncoematologia, Azienda Ospedaliero-

Universitaria di Parma; 'Dipartimento

Materno-Infantile, U.O. Oncoematologia

Pediatrica, Azienda Ospedaliero-

Universitaria Policlinico, Modena;

10Oncematologia Pediatrica San Giovanni

Rotondo; "1S.C. Oncoematologia

Pediatrica, Az. Ospedaliera OIRM S.

Anna, Torino; 12Department of Pediatric

Hematology, San Gerardo Hospital,

Monza, University of Milan-Bicocca,

Milan; ${ }^{13}$ Infectious Diseases Unit,

Department of Hematology and

Oncology, G. Gaslini Children's Hospital, Genoa, Italy

\section{Abstract}

The most intensive chemotherapy regimens were used in the past for leukemia patients who were the main focus of trials on infections; today there are increasing numbers of children with solid cancer and considerable risk of infection who do receive intensive standard-dose chemotherapy. Despite a continuous will to protect the immune-compromised child from infections, evidence-based indications for intervention by non-pharmacological tools is still lacking in the pediatric hematology-oncology literature. Guidelines on standard precautions as well as precautions to avoid transmission of specific infectious agents are available. As a result of a consensus discussion, the Italian Association for Pediatric Hematology-Oncology (AIEOP) Cooperative Group centers agree that for children treated with chemotherapy both of these approaches should be implemented and vigorously enforced, while additional policies, including strict environmental isolation, should be restricted to patients with selected clinical conditions or complications. We present here a study by the working group on infectious diseases of AIEOP.

\section{Introduction}

In the past the most intensive chemotherapy regimens were used for leukemia patients who were the main subjects for trials on infections, and today there is an increasing number of children with solid cancer who are at considerable risk for infection and who receive intensive standard-dose chemotherapy.

The aim of non-pharmacological prophylaxis is to reduce the risk of transmission of infection to the patient as much as possible while he/she is in the ward. This has been the object of specific guidelines by the Centers for Disease Control (CDC), published in 2007.2 However, these guidelines are general recommendations for any hospital environment and not specific for the immunocompromised host, particularly the pediatric oncology patient. Furthermore, most recommendations are based on uncontrolled studies or experts' opinions rather than on randomized controlled trials, and they frequently refer to patients undergoing hematopoietic stem cell transplantation (HSCT), while no specific recommendations are available for patients treated with chemotherapy alone. ${ }^{3}$

In an attempt to provide uniform criteria in this field, the Italian Association for Pediatric Hematology-Oncology (AIEOP) organized a consensus conference at which the issue was debated. We present a paper by the working group on infectious diseases of AIEOP, which includes suggestions of standard precautions for any patient (Table 1), and of solutions to prevent specific transmission-based infections (Table 2).
Correspondence: Désirée Caselli, Dipartimento Oncoematologia Pediatrica, Azienda 0spedalieroUniversitaria Meyer, Firenze.

E-mail: d.caselli@meyer.it

Key words: leukemia, infections, chemotherapy, pediatrics.

Received for publication: 7 December 2010. Accepted for publication: 16 March 2011

This work is licensed under a Creative Commons Attribution 3.0 License (by-nc 3.0)

(C) Copyright D. Caselli et al., 2011

Licensee PAGEPress, Italy

Pediatric Reports 2011; 3:e9

doi:10.4081/pr.2011.e9

\section{Background knowledge}

Several host factors can increase susceptibility to infection, including the following: a variety of medications that alter the normal flora (e.g. antimicrobial agents, gastric acid suppressants, corticosteroids, immune suppressive drugs, antineoplastic agents, and immunosuppressive drugs); surgical procedures and radiation therapy that impair defenses of the skin and other involved organ systems; indwelling devices such as central venous (or arterial) catheters, which facilitate development of infections by allowing potential pathogens to bypass local defenses. This would ordinarily impede their invasion, and provide surfaces for development of bio-films that may facilitate adherence of microorganisms and protect from antimicrobial activity. ${ }^{4-6}$ Children with cancer may have additional risk factors owing to their age and age-related behaviors: an increased numbers of close physical contacts between them and healthcare personnel may occur because of cuddling, feeding, playing, changing soiled diapers, and cleaning copious uncontrolled respiratory secretions. This may provide abundant opportunities for transmission of infectious material. Practices and behaviors such as congregation of children in play areas where toys and body secretions are easily shared, and family members rooming in can further increase the risk of transmission. Pathogenic bacteria have been recovered from toys used by hospitalized patients; contaminated bath toys were implicated in an outbreak of multidrug-resistant Pseudomonas aeruginosa in a pediatric oncology unit. ${ }^{7}$ Pathogen outbreak is a major concern in the healthcare setting, especially for resistant strains.2,3 Is physical isolation a response to this worry? A randomized trial among patients with acute leukemia, performed in 1978, showed a decrease in fatal infections with increased survival among patients treated in a protected environment with prophylactic antibiotics. ${ }^{8}$ However, even 
in these early studies, the independent role of isolation versus antibiotic prophylaxis was not addressed, and the recognition of emerging antibiotic-resistant strains of bacteria when using antibiotics for patient protection rapidly became a worry. ${ }^{9}$ Thus, available knowledge in this field is very limited and mostly derived from experience in patients undergoing HSCT, incorporating the use of laminar air flow (LAF) units, patient isolation units, prophylactic antibiotics, sterile and low-microbial diets, and antimicrobial decontamination. ${ }^{10-13}$ Even in this setting, early prospective, randomized studies of protective environments among HSCT recipients suggested that LAF isolation and decontamination procedures resulted in a significant reduction in infections, but in no difference in survival, with most deaths a result of interstitial pneumonia or recurrent disease..$^{10}$ A retrospective study of the use of HEPA filtration units showed a reduction in the number of Aspergillus organisms in the air and a decrease in the risk of nosocomial Aspergillus infections. ${ }^{14}$

\section{Current AIEOP standards}

The AIEOP cooperative group has been working in pediatric hematology-oncology for about 40 years. ${ }^{15}$ On the basis of the growing experiece in the treatment of children with cancer, constitutional or acquired bone marrow failure syndromes, or immune deficiencies, special attention has been paid to all measures aimed at reducing the risk for fatal infectious complications. Most of the effort has been aimed at developing pharmacological protocols for treatment or even prophylaxis of infections in the immune compromised host. ${ }^{16}$ Cooperative protocols and guidelines for supportive therapy have been developed mostly as part of the chemotherapy treatment protocols. However, no special recommendations have been developed for non-pharmacological prophylaxis to date. As a general rule, isolation of patients at higher risk for infection, and in particular during HSCT, has been applied. During the mid 1980s, the use of LAF hoods became popular, especially among the larger centers. ${ }^{13}$ Otherwise, no specific regulations or recommendations have been generated by the association. Thus, current policies in the over 40 AIEOP centers are based on local experience or attitudes. This prompted the group to address the issue of the opportunity to standardize the behaviors in the participant centers.

\section{Measures aimed at protection of the patient}

Overall, several issues have to be considered when trying to define if and which measures of non-pharmacological methods are indicated for prevention of infection in children undergoing chemotherapy. Available indications are sparse, mainly derived from observation of

Table 1. Standard precautions.*

Handwashing (or using an antiseptic handrub)

- After touching blood, body fluids, secretions, excretions, and contaminated items

- Immediately after removing gloves

- Between patient contacts

Gloves

- For contact with blood, body fluids, secretions, and contaminated items

- For contact with mucous membranes and non-intact skin

Masks, goggles, face masks

- Protect mucous membranes of eyes, nose, and mouth when contact with blood and body fluids is likely

Gowns

- Protect skin from blood or body fluid contact

- Prevent soiling of clothing during procedures that may involve contact with blood or body fluids

Linen

- Handle soiled linen to prevent touching skin or mucous membranes

- Do not pre-rinse soiled linen in patient care areas

Patient care equipment

- Handle soiled equipment in a manner to prevent contact with skin or mucous

membranes and to prevent contamination of clothing or the environment

- Clean reusable equipment prior to reuse

Environmental cleaning

- Routinely care, clean, and disinfect equipment and furnishings in patient care areas

Sharps

- Avoid recapping used needles

- Avoid removing used needles from disposable syringes

- Avoid bending, breaking, or manipulating used needles by hand

- Place used sharps in puncture-resistant containers

Patient resuscitation

- Use mouthpieces, resuscitation bags, or other ventilation devices to avoid mouth-to-mouth resuscitation

Patient placement

- Place patients who contaminate the environment or cannot maintain appropriate hygiene in private rooms

*Adapted from Ljungman et al., 2009.

patients undergoing transplants, and never obtained through specific studies or even prospective data collection. Recommendations have focused on issues of ventilation, construction, room cleaning, isolation and barrier precautions, interactions with healthcare workers and visitors, skin and oral care, infection surveillance, and prevention of specific nosocomial and seasonal infections.

In an era of increasing financial constraints, recommendations on specific interventions that may turn out to be expensive and timeconsuming need to be carefully revisited and, whenever possible, validated. Regarding this issue, it may be interesting to note that while the CDC/IDSA/ASBMT guidelines contain over 200 recommendations for infection control among HSCT recipients, only seven are supported by level I (randomized trial) evidence. Six recommendations hold clinical benefit and one is associated with harm. These level I recommendations do not include the use of patient isolation units, ventilation systems, construction or cleaning guidelines, skin or oral care, or the prevention of catheter-associated infections. The first $\mathrm{AI}$ recommendation (strongly recommended with randomized trial support) involves the long-held, low-technology, infection control practice of handwashing. ${ }^{2}$ Guidelines for hand hygiene have been published, which specifically address issues of the indications for handwashing and antisepsis, the handwashing technique, surgical hand antisepsis, the selection of hand hygiene agents, and even healthcare worker educational and motivational programs. ${ }^{17}$ However, a cross-sectional survey of university hospital physicians showed a dismal 57\% average adherence. ${ }^{18}$ Concerning the handwashing policy, the problem is how to improve this attitude and its compliance in the hospitals. Many studies suggest that the education and controls must be continuous, to highlight the attention of careworkers to this topic: easy, timely access to both hand hygiene and skin protection is necessary for satisfactory hand hygiene behavior. Alcohol-based hand rubs may be better than traditional handwashing as they require less time, act faster, are less irritating, and contribute to sustained improvement in compliance associated with decreased infection rates. ${ }^{19}$ Whenever possible, local surveys on 
Table 2. Transmission-based precautions are used when the route(s) of transmission is(are) not completely interrupted using standard precautions alone.*

\section{Airborne precautions}

Used in addition to standard precautions for a patient known or suspected to be infected with microorganisms transmitted by the airborne route

\section{Droplet precautions}

Used in addition to standard precautions for a patient known or suspected to be infected with microorganisms transmitted by large-particle droplets (larger than $5 \mu \mathrm{m}$ )

\section{Contact precautions}

Used in addition to standard precautions

for a patient known or suspected to be infected or colonized with microorganisms transmitted by direct contact with the patient or indirect contact with environmental surfaces or patient care items

\section{Patient placement}

Door closed

Room air is exhausted to the outside (negative air pressure) using fan or other filtration system

If private room is not available, place patient in room with patient with active infection with the same disease, but with no other infection

If TB is known or suspected, wear particulate respirator (if available)

If chickenpox or measles: for immune persons, no mask is required;

susceptible persons, do not enter room Remove PPE (face shield) after leaving the room and place it in a plastic bag or waste container with tight-fitting lid

\section{Patient placement}

Private room,

door may be left open

If private room is not available, place patient in room with patient with active infection with the same disease, but with no other infection

If neither option is available, maintain separation of at least $3 \mathrm{ft}$ between patients

Respiratory protection

Wear mask if within $3 \mathrm{ft}$ of patient

\section{Patient placement}

Private room, door may be left open

If private room is not available, place patient in room with patient with active infection with the same microorganism, but with no other infection

\section{Contact protection}

\section{Gloving}

Wear clean, nonsterile examination gloves when entering room

Change gloves after contact with infective material (e.g. fecal materials or wound drainage)

Remove gloves before leaving patient's room Handwashing

Wash hands with antibacterial agent or use alcohol-based handrub after removing gloves Do not touch potentially contaminated surfaces or items before leaving the room

Gowns and protective apparel

Wear clean, nonsterile gown when entering patient's room if you anticipate contact with patient or if the patient is incontinent, has diarrhea, an ileostomy, colostomy, or wound drainage not contained by a dressing Remove gown before leaving room. Do not allow clothing to contact potentially contaminated surfaces or items before leaving room

Patient transport Patient transport

Limit transport of patient to essential purposes only. During transport, patient must wear surgical mask

Notify area receiving patient
Limit transport of patient to essential purposes only

During transport, patient must wear surgical mask. Notify area receiving patient

\section{Patient transport}

Limit transport of patient to essential purposes only During transport, ensure precautions are maintained to minimize risk of transmission of organisms Patient care equipment

Reserve noncritical patient care equipment for use with a single patient, if possible

Clean and disinfect any equipment shared among infected and noninfected patients

*Adapted from Ljungman et al., 2009.

Table 3. Empiric use of transmission-based precautions.*

\begin{tabular}{lll}
\hline Airborne & Droplet & Contact \\
Rashes (vesicle or pustule) & Severe, persistent cough during periods & Acute diarrhea in an incontinent or diapered patient \\
$\begin{array}{l}\text { Cough, fever, and upper lobe chest findings } \\
\text { (dullness and decreased breath sounds) }\end{array}$ & when pertussis is present in community & Diarrhea with history of recent antibiotic use \\
& Generalized rash of unknown cause & $\begin{array}{l}\text { Bronchitis and croup } \\
\text { History of infection with multi-resistant organisms }\end{array}$ \\
& & Abscess or draining wound that cannot be covered \\
\hline
\end{tabular}

*Adapted from Ljungman et al., 2009 . 
the compliance promoted by the hospital authority may support the continuous applications of these measures. The CDC/IDSA/ ASBMT guidelines address an additional 12 recommendations graded AII (strongly recommended with well-designed non-randomized trial support). While the utility of specific isolation and barrier precautions have not been studied, a level AIII recommendation (strongly recommended with expert opinion support) is given to follow other published guidelines for hospital isolation and the prevention of nosocomial infections such as pneumonia or surgical site infections. ${ }^{2}$

Measures aimed at protection

of contact persons, especially

caregivers

All suggestions aimed at protecting caregivers are included in the universal precautions section.

Contact with animals and avoiding foodborn infections

These issues were not addressed in the consensus.

Measures aimed at preventing transmission of specific agents according to their route of spread

A relevant issue may be the choice of which strategy we may want to adopt to prevent dissemination of specific infections according to their usual route of spread. Thus, additional infection control recommendations can be considered for epidemiologic factors such as the host, human-to-human interactions, fomites, air, food, water, soil, and construction and cleaning (Table 2). These precautions, which are aimed at limiting the spread of infectious agents and designed for avoiding specific infections, should be applied empirically, according to the clinical condition and the likely etiologic agents at the time, and then modified when either the pathogen is identified or a transmissible infectious etiology is ruled out (Table 3). The clinician's experience will direct specific restrictions for patients for whom multidrug-resistant bacterial strains or other potentially dangerous exposure(s) may reasonably be suspected.

\section{Conclusions}

In conclusion, despite the general attitude toward children undergoing chemotherapy, and particularly the practice of isolating them, there may be a wide range of inter-center variability. Most likely, isolation of individual children cannot be justified by currently available data, unless they have specific complications rather than uncomplicated fever of unknown origin. The use of air filtering or other environmental modifications, although possibly not incorrect, cannot be considered as the desirable standard of care. On the contrary, standard procedures, such as those suggested by CDC, appear warranted and definitely need to be enforced. As a paradigm, repeated and careful handwashing remains a pivotal behavior in limiting the horizontal spread of infectious agents among children in a pediatric hematology-oncology ward. As a result of the meeting, the expert opinion of AIEOP's panel recommends using a combined approach of standard and transmission-based precautions for children undergoing chemotherapy while additional, more sophisticated and expensive environmental procedures or structures cannot be considered as mandatory.

\section{References}

1. Ljungman P, Bregni $M$, Brune $M$, et al. Allogeneic and autologous transplantation for haematological diseases, solid tumours and immune disorders: current practice in Europe 2009. Bone Marrow Transplant 2010;45:219-34.

2. Siegel JD, Rhinehart E, Jackson M, Chiarello L, and the Healthcare Infection Control Practices Advisory Committee, 2007. Guideline for isolation precautions: preventing transmission of infectious agents in healthcare settings. Address: http://www.cdc.gov/ncidod/dhqp/pdf/isolation2007.pdf

3. Hayes-Lattin B, Leis JF, Maziarz RT. Isolation in the allogeneic transplant environment: how protective is it? Bone Marrow Transplant 2005;36:373-81.

4. Simon A, Ammann RA, Bode U, et al. Healthcare-associated infections in pediatric cancer patients: results of a prospective surveillance study from university hospitals in Germany and Switzerland. BMC Infect Dis 2008;8:70.

5. Castagnola E, Fontana V, Caviglia I, et al. A prospective study on the epidemiology of febrile episodes during chemotherapyinduced neutropenia in children with cancer or after hemopoietic stem cell transplantation. Clin Infect Dis 2007;45:1296304.

6. Oberdorfer P, Pongwilairat N, Washington $\mathrm{CH}$. Nosocomial infections among pediatric patients with neoplastic diseases. Int $\mathrm{J}$ Pediatr 2009;2009:721320.

7. Buttery JP, Alabaster SJ, Heine RG, et al. Multiresistant pseudomonas aeruginosa outbreak in a pediatric oncology ward related to bath toys. Pediatr Infect Dis J 1998;17:509-13.

8. Rodriguez V, Bodey GP, Freireich EJ, et al. Randomized trial of protected environment - prophylactic antibiotics in 145 adults with acute leukemia. Medicine (Baltimore) 1978;57:253-66.

9. Klastersky J, Debusscher L, Weerts D, Daneau D. Use of oral antibiotics in protected units environment: clinical effectiveness and role in the emergence of antibiotic-resistant strains. Pathol Biol (Paris) 1974;22:5-12.

10. Buckner CD, Clift RA, Sanders JE, et al. Protective environment for marrow transplant recipients: a prospective study. Ann Intern Med 1978;89:893-901.

11. Storb R, Prentice RL, Buckner CD, et al. Graft-versus-host disease and survival in patients with aplastic anemia treated by marrow grafts from HLA-identical siblings. Beneficial effect of a protective environment. N Engl J Med 1983;308:302-7.

12. Beelen DW, Elmaagacli A, Muller KD, et al. Influence of intestinal bacterial decontamination using metronidazole and ciprofloxacin or ciprofloxacin alone on the development of acute graft-versus-host disease after marrow transplantation in patients with hematologic malignancies: final results and longterm follow-up of an open-label prospective randomized trial. Blood 1999;93:3267-75.

13. Passweg JR, Rowlings PA, Atkinson KA, et al. Influence of protective isolation on outcome of allogeneic bone marrow transplantation for leukemia. Bone Marrow Transplant 1998;21:1231-8.

14. Sherertz RJ, Belani A, Kramer BS, et al. Impact of air filtration on nosocomial Aspergillus infections. Unique risk of bone marrow transplant recipients. Am J Med 1987;83:709-18

15. Paolucci G, Masera G, Vecchi V, et al. Treating childhood acute lymphoblastic leukaemia (ALL): summary of ten years' experience in Italy. ALL Steering Committee of the Associazione Italiana Ematologia Oncologia Pediatrica (AIEOP). Med Pediatr Oncol 1989;17:83-91.

16. Viscoli C, Castagnola E, Caniggia M, et al. Italian guidelines for the management of infectious complications in pediatric oncology: empirical antimicrobial therapy of febrile neutropenia. Oncology 1998;55:489-500.

17. Centers for Disease Control and Prevention. Guideline for hand hygiene in health-care settings: recommendations of the Healthcare Infection Control Practices Advisory Committee and the HICPAC/ SHEA/APIC/IDSA Hand Hygiene Task Force. MMWR 2002;51:RR1-45.

18. Pittet D, Simon A, Hugonnet S, et al.Guidelines for hand hygiene among physicians: performance, beliefs, and perceptions. Ann Intern Med 2004;141:1-8.

19. Pittet D. Improving adherence to hand hygiene practice: a multidisciplinary approach. Emerg Infect Dis 2001;7:234-40. 